\title{
Clinical Relevance of Cluster Analysis in Phenotyping Allergic Rhinitis in a Real-Life Study
}

\author{
Philippe Jean Bousquet $^{\mathrm{a}} \quad$ Philippe Devillier $^{\mathrm{b}} \quad$ Abir Tadmouric $^{\mathrm{c}}$ Kamal Mesbah $^{\mathrm{d}}$ \\ Pascal Demoly ${ }^{a, e}$ Jean Bousquet ${ }^{a, f}$ \\ a University Hospital, and INSERM CESP 1018, Montpellier, b UPRES EA 220, Hôpital Foch, Université de Versailles Saint \\ Quentin, Suresnes, 'Scientific and Medical Department, ClinSearch, Bagneux, dMSD France, Courbevoie, ${ }^{\mathrm{d}}$ Département \\ de Pneumologie et Allergologie, Hôpital Arnaud de Villeneuve, and INSERM U657, CHU de Montpellier, Montpellier, \\ and ${ }^{\mathrm{f}}$ Department of Respiratory Diseases, University Hospital, and INSERM U1018, Paris, France
}

\section{Key Words}

Allergic rhinitis - Unsupervised cluster analysis · ARIA .

Uncontrolled allergic rhinitis · Stratification

\begin{abstract}
Background: Disease stratification, using phenotypic characterization performed either by hypothesis- or data-driven methods, was developed to improve clinical decisions. However, cluster analysis has not been used for allergic rhinitis. Objective: To define clusters in allergic rhinitis and to compare them with ARIA (Allergic Rhinitis and Its Impact on Asthma), a hypothesis-driven approach. Methods: A French observational prospective multicenter study (EVEIL: Echelle visuelle analogique dans la rhinite allergique) was carried out on 990 patients consulting general practitioners for allergic rhinitis and treated as per clinical practice. In this study, changes in symptom scores, visual analogue scales and quality of life were measured at baseline and after 14 days of treatment. A post hoc analysis was performed to identify clusters of patients with allergic rhinitis - using Ward's hierarchical method - and to define their clinical relevance at baseline and after 14 days of treatment. The cluster approach was compared to the ARIA approach. Results: Patients were clustered into 4 phenotypes which partly followed the ARIA
\end{abstract}

classes. These phenotypes differed in their disease severity including symptoms and quality of life. Physicians in real-life practice prescribed medication regardless of the phenotype and severity, with the exception of patients with ocular symptoms. Prescribed treatments were comparable in hypothesis- and data-driven analyses. The prevalence of uncontrolled patients during treatment was similar in the 4 clusters, but was significantly different according to the ARIA classes. Conclusion: Cluster analysis using demographic and clinical parameters only does not appear to add relevant information for disease stratification in allergic rhinitis.

(c) 2015 S. Karger AG, Basel

\section{Introduction}

Noncommunicable chronic diseases, such as chronic respiratory diseases, are generally complex and heterogeneous. Disease stratification, using phenotypic characterization which can be performed either by hypothesis- or data-driven methods, was developed to improve clinical decisions [1]. The endotype concept was proposed by Anderson [2]. This definition has been used by others: 'An endotype is a subtype of asthma defined by a distinct pathophysiological mechanism'.

\section{KARGER 125}

(c) 2015 S. Karger AG, Base

$1018-2438 / 15 / 1663-0231 \$ 39.50 / 0$

E-Mail karger@karger.com

www.karger.com/iaa
Correspondence to: Prof. Jean Bousquet

Département Pneumologie et Addictologie CHU Montpellier

FR-34295 Montpellier Cedex 05 (France)

E-Mail jean.bousquet@inserm.fr 
The hypothesis-driven stratification of patients with allergic rhinitis (AR) generally embeds clinical outcomes, disease severity or control [3]. No objective parameters or biomarkers have been identified to stratify patients with AR. ARIA (Allergic Rhinitis and Its Impact on Asthma), the most widely used guideline, includes symptom severity, quality of life (QoL) and duration of disease, and proposes a simple stratification of AR applicable in daily practice [4]. This classification can be used in treated or untreated patients [5]. The visual analogue scale (VAS) has been used in many studies [6-9] and was found to be clinically relevant for stratifying patients and assessing treatment efficacy $[3,5,10,11]$. Patients who fail to respond to pharmacologic treatment are classified as SCUAD (severe chronic upper airway disease) [12, 13].

A novel phenotypic characterization for allergic diseases appears to be needed to distinguish groups of patients presenting homogeneous clinical, prognostic characteristics and responses to treatments. The identification of phenotypes could lead to an improved management and AR control. Popular epidemiologic approaches, such as the unsupervised statistical techniques, have recently emerged to meet these needs. These approaches consist of applying unsupervised statistical methods to a population with a wide distribution of related symptoms, and then identifying the possible underlying phenotypes. Each of these phenotypes should be as homogeneous as possible and have as little overlap as possible with each of the other phenotypes. Phenotypes are not known a priori, and there are no predefined views about them. There are different types of clustering approaches such as hierarchical, partitioning and model-based clustering [14] or network analysis [15]. Many studies in asthma and allergic diseases have used various clustering methods to stratify patients [16-22]. One of the recommended methods is Ward's hierarchical cluster analysis [23, 24].

To our knowledge, these data-driven approaches have not been published in a patient population suffering from AR. The validation of the observed phenotypes is a critical and yet unresolved issue that may be performed by testing the predictive performance of clusters in a clinical trial. The clinical benefit of using cluster analysis versus a hypothesis-driven approach has not been established. Moreover, it may be difficult to apply a cluster analysis in clinical practice.

A French observational prospective multicenter study (EVEIL: Echelle visuelle analogique dans la rhinite allergique) was carried out on 990 patients consulting general practitioners (GPs) for AR and treated as per clinical practice. In this study, changes in symptom scores, VAS and QoL were measured at baseline and after 14 days of treatment [25]. The study involved a careful evaluation of demographic and clinical characteristics [26] and was suitable for cluster analysis. We performed a post hoc analysis using the dataset of the EVEIL study to identify clusters of patients with AR using Ward's hierarchical method, and to define their clinical relevance at baseline and after 14 days of treatment. The cluster approach was compared to ARIA, a hypothesis-driven approach largely used in clinical practice [4].

\section{Methods}

\section{Study Design}

The data were obtained from a multicenter prospective observational study carried out throughout France (EVEIL). The protocol and descriptive characteristics are described elsewhere $[25,26]$. The main objective of this post hoc analysis was to identify clusters using Ward's method. Secondary objectives were (1) to compare VAS, QoL, total symptom scores (TSS) and ARIA classes at baseline in the identified clusters, (2) to define the treatments received by the patients and their efficacy according to the clusters, (3) to compare the differences in patient stratification and prediction of treatment benefits by using cluster analysis (data driven), ARIA guidelines (hypothesis driven) or the levels of VAS at baseline. The percentage of uncontrolled patients (SCUAD) was also assessed in each cluster or ARIA class.

GPs were randomly selected by means of geographic stratification. Physicians completed a questionnaire on the consultation day which included sociodemographic data, diagnostic history, rhinitis symptoms, concomitant diagnosed pathologies and treatment prescription. The management of AR was at the physicians' discretion and patients were reviewed after 14 days of treatment.

\section{Patients}

Patients were included in the study after oral informed consent was obtained and after approval of the protocol by the Ethics Committee of Montpellier, France. Patients were enrolled for 1 year (February 2010 to January 2011) and were selected from all regions of France in order to rule out any geographic or seasonal parameter. All patients fulfilled the following inclusion criteria: over 18 years of age with a diagnosis of AR and presenting clinical symptoms of AR on the consultation day.

\section{Outcome Measures}

Individual nasal and ocular symptoms, TSS, Rhinoconjunctivitis Quality of Life Questionnaire (RQLQ) and VAS were assessed by the patients on the consultation day and 14 days later (online suppl. S1; for all online suppl. material, see www.karger.com/ doi/10.1159/000381339).

\section{Cluster Analysis}

Variable Reduction

The whole dataset provided many variables that required reduction in number before performance of a cluster analysis. The selection of input variables was processed by excluding irrelevant 
variables, variables with many missing data ( $>100$ missing data) and variables with text format (e.g. 'others'; online suppl. fig. S1). For the current analysis, two variables were considered as irrelevant: (1) the physician who diagnosed the disease and (2) the information about the next follow-up consultation (the date). After reduction, relationships between the 18 variables were studied by cluster analysis, using the VARCLUS (variable cluster) procedure $[27,28]$. This procedure, which organizes a set of numeric variables into hierarchical clusters, can be used to examine redundancy between variables. Therefore, one single representative variable was chosen from each cluster and the other variables were discarded. It can be noted that the smallest value of the $1-\mathrm{R}^{2}$ ratio was used to guide the selection. A summary of this procedure is presented in online supplementary table S1.

Identification of AR Phenotypes

Ward's minimum-variance hierarchical clustering was performed to identify AR phenotypes. In this method, the agglomerative approach was based on the quantitative measures of similarity procedure (minimum within cluster sum of square). Subsequently, patients in the same cluster were more similar to each other than those in different clusters. The algorithm then proceeded by merging the two most similar clusters until there was only one single cluster. We used pseudo-F statistics to determine the optimal number of clusters in the data.

\section{Biases}

To reduce biases, we performed an unsupervised clustering using a statistical algorithm to select classes inherent to the data. Therefore, the statistical method used allows unbiased analyses that are not based on any a priori assumptions. One of the potential biases would be the population included in the algorithm. Only 825 out of the 990 recruited patients were included in the algorithm. Patients with incomplete datasets were excluded from the analyses, which requested complete data. Clustering was exclusively based on clinical variables and questionnaires - no biological variables or biomarker data were available. Our approach was appropriate for the identification of clusters in clinical practice. It is possible that inclusion of other variables (e.g. biomarkers) may have improved the ability to identify phenotypes.

\section{Statistical Analysis}

Descriptive statistics were supplied according to the variable nature. For quantitative variables, the number of nonmissing observations, arithmetic mean, median, standard deviation, minimum and maximum were presented. For qualitative variables, the number of nonmissing observations, frequency and percentage were presented.

The clusters were compared to identify which of the input variables were significantly different between clusters. Comparisons were provided using ANOVA or a Kruskal-Wallis test for quantitative variables and Pearson's $\chi^{2}$ or Fischer's exact test, as appropriate, for qualitative variables.

The description of real-life management and outcomes was performed on the identified clusters using descriptive statistics. The described variables included VAS, TSS and RQLQ outcomes before and after treatment.

Logistic regression was used to identify factors associated with uncontrolled SCUAD AR. Patients with SCUAD were defined pre hoc as those with a VAS level $\geq 50 \mathrm{~mm}$ and/or presence of ocular symptoms (ranked $\geq 2$ ) at the end of the 2-week treatment [13]. The variables significant at the 0.2 level in bivariate analyses were entered into this multivariate logistic regression.

\section{Results}

\section{Demographic Characteristics}

A total of 191 GPs throughout France participated in the EVEIL study and 161 enrolled at least 1 patient. 990 consecutive patients consulting for AR were included. Among the 990 patients, 825 (83\%) were included in this post hoc analysis (online suppl. fig. S2). Patients with missing data identified during the VARCLUS procedure were excluded. The baseline characteristics of the patients are described in online supplementary S2.

\section{Cluster Description}

The classification of 825 patients with AR using agglomerative cluster analysis generated a dendrogram (online suppl. fig. S3). As is shown in the dendrogram, cluster 1 was differentiated mainly by the treatments received prior to the consultation day such as oral/nasal antihistamine, corticoids, ocular antihistamine and OTC. Cluster 2 was characterized by the severity of the disease assessed using the ARIA classes, VAS and TSS6 at inclusion. Cluster 3 was characterized by the presence of comorbidities such as a documented allergy, presence of concomitant skin or respiratory disease and the use of antileukotriene (antiasthmatic agent), and cluster 4 identified patient age and duration of diagnostics.

Four clusters classified by number of subjects per cluster (1-4) were identified from mild to severe phenotype (fig. 1). Two extreme phenotypes were identified: cluster 2 was the mildest phenotype, whereas cluster 4 was the most severe. Significant differences in age, symptoms and severity were found among the four clusters. Baseline sociodemographics and clinical characteristics of the 825 patients according to these four clusters are presented in table 1 . Symptoms and disease burden at baseline are presented in table 2 .

Clusters 1 and 3 included patients with moderate-tosevere phenotypes. However, cluster 1 was more severe than cluster 3 , with slightly more patients suffering from severe/moderate persistent AR. Compared with patients in cluster 3, patients in cluster 1 had the worst QoL and symptoms, and the greatest disease burden. Cluster 3 was the only cluster where the majority of patients were male.

Cluster 2 consisted mainly of women with a median age of 40 years. The median age of AR onset was 5 years. 


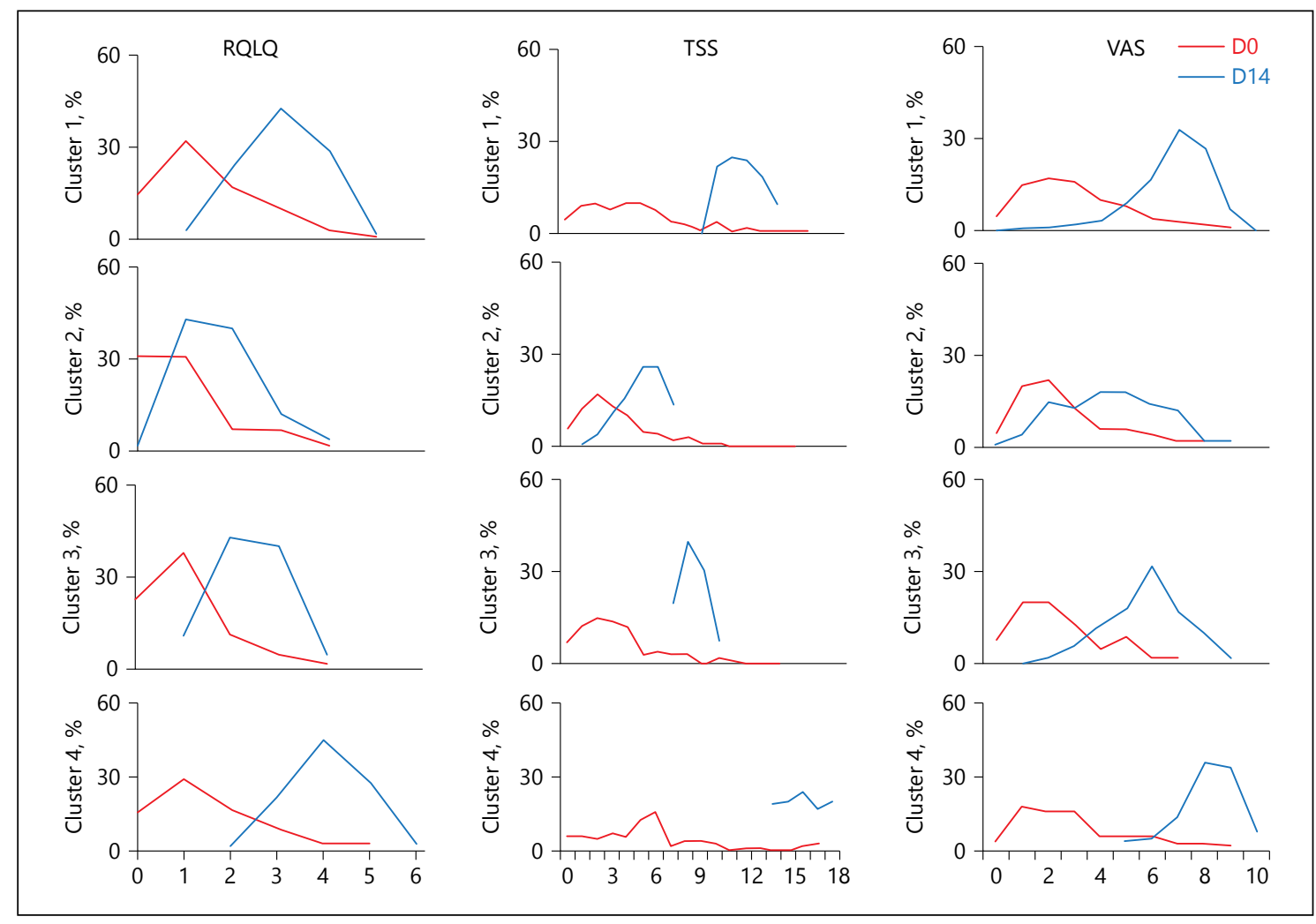

Fig. 1. Distribution of patients according to the scores RQLQ, TSS and VAS at baseline (red) and at day 14 after treatment (blue).

The patients in this cluster were mainly classified as having mild intermittent AR. They had less concomitant comorbidities, including skin or respiratory conditions, than the other clusters. They experienced significantly fewer symptoms than the other clusters. Cluster 2 patients had a mild impairment in QoL, with a mild symptom score and low level of disease burden.

Cluster 4 consisted mainly of young women: $59 \%$ were female patients with a median age of 36 years. The median age of AR onset was 10 years, the oldest between the clusters. The majority of patients in cluster 4 presented with severe/moderate persistent AR (75\%) and with a diagnosed allergy (60\%). Cluster 4 patients had a significantly impaired QoL with a severe symptom score and high level of disease burden.

Differences in some allergens were observed between clusters, particularly for tree pollen, grasses and weeds (table 1).

On the consultation day, $73 \%$ of patients were receiving medications for AR regardless of the cluster or disease severity. Treatment of AR received prior to the GP consultation was not a confounding variable among the clusters.

\section{Real-Life Management and Medication Prescribed during the Consultation}

During the consultation, all patients received a prescription for medications. $73 \%$ received an $\mathrm{H}_{1}$-antihistamine (oral or nasal) associated with corticosteroids $(65 \%$ nasal) (online suppl. table S2). No significant differences were found in the prescription of any treatment between clusters. However, ocular $\mathrm{H}_{1}$-antihistamines and antileukotrienes were more frequently prescribed in clusters 1 and 4 (table 3a).

\section{Clinical Outcomes in Data-Driven Clusters at the End} of Treatment

A significant clinical improvement was observed in all clusters 14 days after consultation with a reduction of TSS, RQLQ and VAS (table 4a). Although there were differences between clusters, they were relatively small.

Twenty-one percent of the patients were characterized as having SCUAD at day 14. A nonsignificant difference was found between the clusters, although clusters $4(30.1 \%)$ and $1(24.4 \%)$ included more SCUAD patients than clusters $2(19.4 \%)$ and $3(15.4 \%)$. 
Table 1. Baseline sociodemographics and clinical characteristics

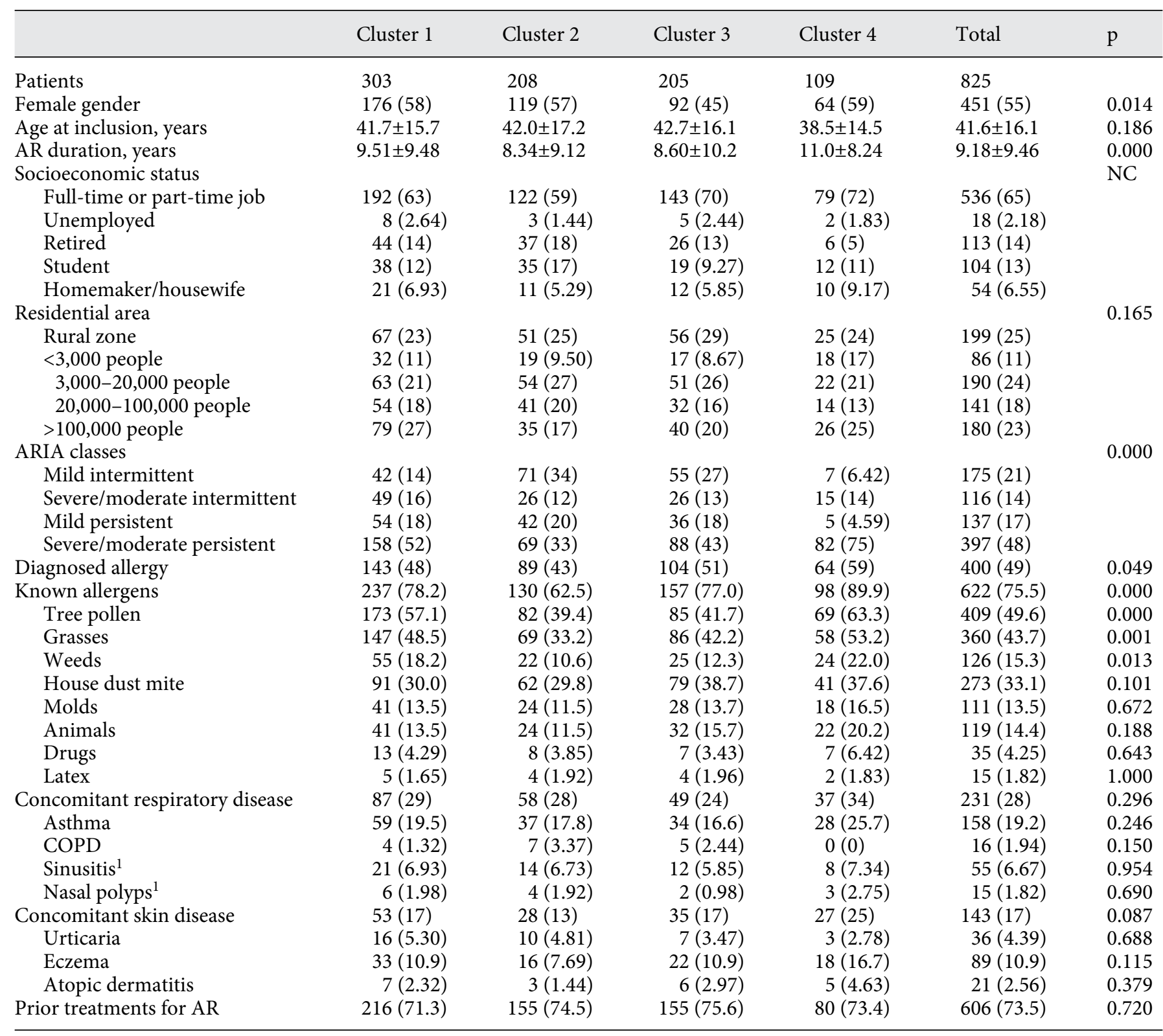

Values are given as means \pm SD or $\mathrm{n}(\%) . \mathrm{NC}=$ Not computed; COPD $=$ chronic obstructive pulmonary disease.${ }^{1}$ Sinusitis included the acute and chronic forms. Sinusitis and nasal polyps were reported at the physician's discretion. No definitions were provided in the protocol.

Clinical Outcomes in Hypothesis-Driven ARIA Classes at the End of Treatment

Patients classified by ARIA at baseline were prescribed a similar treatment in the 4 classes except for ocular $\mathrm{H}_{1}$-antihistamines that were prescribed more often in patients with ocular symptoms (table $3 \mathrm{~b}$ ). The percentage of SCUAD patients after 14 days of treat- ment was significantly different depending on ARIA classes, ranging from $9.9 \%$ in mild intermittent rhinitis to $27.9 \%$ in moderate/severe persistent rhinitis. Similarly, TSS6, RQLQ and VAS levels after treatment increased significantly from mild intermittent rhinitis to mild persistent, moderate-severe intermittent and moderate-severe persistent rhinitis (table $4 \mathrm{~b}$ ). 
Table 2. Symptoms and rhinitis burden at baseline

\begin{tabular}{|c|c|c|c|c|c|c|}
\hline Patients, $\mathrm{n}$ & 303 & 208 & 205 & 109 & 825 & \\
\hline Nasal obstruction & $258(85.1)$ & $152(73.4)$ & $169(82.4)$ & $100(91.7)$ & $679(82.4)$ & 0.000 \\
\hline Rhinorrhea & $279(92.4)$ & $176(85.0)$ & $182(88.8)$ & $102(93.6)$ & $739(89.8)$ & 0.026 \\
\hline Nasal pruritus & $239(78.9)$ & $101(48.8)$ & $137(67.2)$ & $98(89.9)$ & $575(69.9)$ & 0.000 \\
\hline Sneezing & $273(90.1)$ & $134(64.7)$ & $173(84.4)$ & $100(91.7)$ & $680(82.5)$ & 0.000 \\
\hline Loss of smell & $153(50.5)$ & $74(35.9)$ & $82(40.0)$ & $64(58.7)$ & $373(45.3)$ & 0.000 \\
\hline Breathlessness & $125(41.3)$ & $59(28.6)$ & $65(31.7)$ & $68(62.4)$ & $317(38.5)$ & 0.000 \\
\hline Fever & $10(3.33)$ & $7(3.38)$ & $4(1.95)$ & $9(8.26)$ & $30(3.65)$ & 0.061 \\
\hline Eyelid edema & $52(17.3)$ & $12(5.80)$ & $15(7.35)$ & $28(25.7)$ & $107(13.0)$ & 0.000 \\
\hline Asthenia & $151(49.8)$ & $69(33.3)$ & $79(38.7)$ & $76(69.7)$ & $375(45.6)$ & 0.000 \\
\hline QoL (RQLQ) & $3.04 \pm 0.78$ & $1.68 \pm 0.83$ & $2.36 \pm 0.70$ & $4.02 \pm 0.80$ & $2.68 \pm 1.07$ & 0.000 \\
\hline Disease burden (100-mm VAS) & $67.4 \pm 14.8$ & $43.9 \pm 19.6$ & $57.0 \pm 15.1$ & $80.8 \pm 11.0$ & $60.7 \pm 19.8$ & 0.000 \\
\hline
\end{tabular}

Values are given as means \pm SD or $\mathrm{n}(\%)$.

\section{Predictive Factors for SCUAD}

A bivariate model followed by multivariate analysis was used to determine the predictive factors of SCUAD in AR patients (table 5). This analysis showed that presence of fever, other respiratory disease, ocular symptoms or the use of ocular $\mathrm{H}_{1}$-antihistamines are predictive factors of SCUAD. On the other hand, mild intermittent AR, presence of nasal obstruction and pruritus were less associated with SCUAD. Clusters were not associated with SCUAD.

\section{Discussion}

A data-driven analysis showed that patients can be clustered into 4 phenotypes which partly follow ARIA, a hypothesis-driven analysis. These phenotypes differed in their disease severity including symptoms and QoL. Physicians in real-life practice prescribe medication regardless of the phenotype and severity, with the exception of patients with ocular symptoms. Prescribed treatments were comparable in hypothesis- and datadriven analysis. The prevalence of uncontrolled patients during treatment (SCUAD) was similar in the 4 clusters but was significantly different according to ARIA classes.

\section{Limitations}

This post hoc study was not initially designed to define clusters, but clinical parameters would not have differed largely if the study had been planned for cluster analysis.

This analysis is an 'unsupervised analysis clustering'. Unsupervised clustering are types of algorithms that try to find correlations between parameters without any external inputs other than the raw data. Only the algorithm decides to group datasets into clusters that share common properties. We chose this analysis since it was a post hoc analysis; the primary objective of the study was to validate the VAS tool in AR. As it was an observational study, there were many missing data and collected parameters were not clinical or standardized. This was the reason why we decided to do an exploratory 'unsupervised' analysis. The selection of criteria is crucial for any cluster analysis. A large number of clinical outcomes were used, including symptoms, QOL and ARIA classes. Biologic criteria were not included, but (1) it has not been shown that blood eosinophils are highly increased in AR in contradistinction to asthma $[16,18],(2)$ the presence of an allergic sensitization did not significantly change the cluster analysis and (3) clusters are quite well differentiated in the present study, suggesting that other criteria may not have a very large impact. In this analysis, we followed methods used elsewhere for asthma [29]. Ward's mini- 
Table 3. Treatment prescribed at the consultation and clinical outcomes

a According to cluster classes

\begin{tabular}{|c|c|c|c|c|c|c|}
\hline & Cluster 1 & Cluster 2 & Cluster 3 & Cluster 4 & Total & $\mathrm{p}$ \\
\hline Patients & 303 & 208 & 205 & 109 & 825 & \\
\hline \multicolumn{7}{|c|}{ Treatments prescribed at the consultation } \\
\hline Corticoids & $231(76)$ & $158(76)$ & $148(72)$ & $90(83)$ & $627(76)$ & 0.239 \\
\hline Cromoglycate-like drugs & $24(7.92)$ & $11(5.29)$ & $8(3.90)$ & $10(9.17)$ & $53(6.42)$ & 0.164 \\
\hline Ocular $\mathrm{H}_{1}$-antihistamine & $118(38.9)$ & $41(19.7)$ & $43(21.0)$ & $51(46.8)$ & $253(30.7)$ & 0.000 \\
\hline Allergen avoidance & $115(38.0)$ & $88(42.3)$ & $86(42.0)$ & $37(33.9)$ & $326(39.5)$ & 0.405 \\
\hline Allergen immunotherapy & $7(2.33)$ & $3(1.44)$ & $7(3.41)$ & $3(2.75)$ & $20(2.43)$ & 0.592 \\
\hline Sick leaves & $15(4.97)$ & $3(1.45)$ & $5(2.46)$ & $8(7.41)$ & $31(3.78)$ & 0.026 \\
\hline Referred to a specialist & $39(13.0)$ & $21(10.1)$ & $22(10.7)$ & $13(11.9)$ & $95(11.5)$ & 0.762 \\
\hline
\end{tabular}

b According to ARIA classes

\begin{tabular}{|c|c|c|c|c|c|c|}
\hline & $\begin{array}{l}\text { Mild } \\
\text { intermittent }\end{array}$ & $\begin{array}{l}\text { Severe/moderate } \\
\text { intermittent }\end{array}$ & $\begin{array}{l}\text { Mild } \\
\text { persistent }\end{array}$ & $\begin{array}{l}\text { Severe/moderate } \\
\text { persistent }\end{array}$ & Total & $\mathrm{p}$ \\
\hline Number of patients & 197 & 150 & 166 & 466 & 979 & \\
\hline \multicolumn{7}{|c|}{ Treatments prescribed at the consultation } \\
\hline Nasal/oral $\mathrm{H}_{1}$-antihistamine & $180(91.4)$ & $140(93.3)$ & $157(94.6)$ & $441(94.6)$ & $918(93.8)$ & 0.428 \\
\hline Corticoids & $141(71.6)$ & $112(74.7)$ & $116(69.9)$ & $364(78.1)$ & $733(74.9)$ & 0.115 \\
\hline Cromoglycate-like drugs & $15(7.61)$ & $13(8.67)$ & $12(7.23)$ & $30(6.44)$ & $70(7.15)$ & 0.815 \\
\hline Decongestant & $47(23.9)$ & $29(19.3)$ & $29(17.5)$ & $92(19.7)$ & $197(20.1)$ & 0.469 \\
\hline Antileukotriene & $7(3.55)$ & $7(4.67)$ & $15(9.04)$ & $39(8.37)$ & $68(6.95)$ & 0.063 \\
\hline Allergy avoidance & $81(41.1)$ & $50(33.3)$ & $69(41.6)$ & $189(40.6)$ & $389(39.7)$ & 0.378 \\
\hline Allergen immunotherapy & $2(1.02)$ & $3(2.00)$ & $4(2.42)$ & $14(3.02)$ & $23(2.36)$ & 0.516 \\
\hline
\end{tabular}

Values are given as $\mathrm{n}(\%)$.

mum-variance hierarchical clustering method was performed using an agglomerative approach and Ward's linkage (dendrogram). Stepwise discriminant analysis was performed to identify the 18 discriminant variables. Using the VARCLUS cluster analysis in SAS, the variables were classified. When the patterns of response to two variables were similar for most patients, these variables were grouped and the variable with the smallest ration $1-\mathrm{R}^{2}$ in the group was selected. Six variables were then selected and considered as discriminant (online suppl. table S1).

EVEIL is a cross-sectional study and clusters can also be defined in longitudinal studies [19, 21]. In children, trajectories of allergic diseases or asthma show that longitudinal-based clusters are of interest [30, 31]. In adults with $\mathrm{AR}$, differences in disease prevalence exist in longi- tudinal studies and patients with AR may develop asthma [32]. However, these changes appear more limited than in children and the transition from AR to AR and asthma is not very common. However, more studies are needed to determine the impact of time on cluster analyses.

It might have been useful to perform several types of cluster analyses since there are different types of approaches like hierarchical, partitioning and model-based clustering [14] or network analysis [15]. However, except for latent class analysis, results are expected to be similar.

It may be considered that the cohort of patients is insufficiently defined and that not all of the criteria have been used (e.g. serum IgE, eosinophils). However, the strength of the study was studying patients who were attending primary care using the criteria GPs use. Not all patients had a diagnosis of allergy, but they are represen- 
Table 4. Clinical outcomes after 14 days of treatment

a Clinical outcomes of patients according to identified clusters

\begin{tabular}{|c|c|c|c|c|c|c|}
\hline & Cluster 1 & Cluster 2 & Cluster 3 & Cluster 4 & Total & $\mathrm{p}$ \\
\hline Patients & 303 & 208 & 205 & 109 & 825 & \\
\hline \multicolumn{7}{|l|}{ Patient assessments at day 14} \\
\hline TSS (TSS6) & $4.83 \pm 3.64$ & $3.47 \pm 2.95$ & $3.52 \pm 2.86$ & $5.54 \pm 3.97$ & $4.25 \pm 3.43$ & 0.000 \\
\hline QoL (RQLQ) & $1.45 \pm 1.05$ & $1.01 \pm 0.92$ & $1.03 \pm 0.89$ & $1.54 \pm 1.25$ & $1.25 \pm 1.04$ & 0.000 \\
\hline Disease burden (100-mm VAS) & $29.5 \pm 19.5$ & $26.8 \pm 19.9$ & $24.8 \pm 18.3$ & $30.1 \pm 21.9$ & $27.7 \pm 19.7$ & 0.039 \\
\hline Uncontrolled AR at day $14^{1}$ & $59(24.4)$ & $33(19.4)$ & $26(15.4)$ & $22(25.6)$ & $140(21.0)$ & 0.101 \\
\hline
\end{tabular}

b Clinical outcomes of patients according to ARIA classes

\begin{tabular}{|c|c|c|c|c|c|c|}
\hline & $\begin{array}{l}\text { Mild } \\
\text { intermittent }\end{array}$ & $\begin{array}{l}\text { Severe/moderate } \\
\text { intermittent }\end{array}$ & $\begin{array}{l}\text { Mild } \\
\text { persistent }\end{array}$ & $\begin{array}{l}\text { Severe/moderate } \\
\text { persistent }\end{array}$ & Total & $\mathrm{p}$ \\
\hline Patients & 197 & 150 & 166 & 466 & 979 & \\
\hline TSS (TSS6) & $3.31 \pm 3.14$ & $4.21 \pm 3.91$ & $4.04 \pm 3.39$ & $5.01 \pm 3.67$ & $4.39 \pm 3.62$ & 0.000 \\
\hline QoL (RQLQ) & $0.93 \pm 0.86$ & $1.25 \pm 1.14$ & $1.17 \pm 1.00$ & $1.48 \pm 1.11$ & $1.29 \pm 1.08$ & 0.000 \\
\hline Disease burden (100-mm VAS) & $21.7 \pm 16.2$ & $28.2 \pm 20.6$ & $26.1 \pm 19.0$ & $31.9 \pm 21.4$ & $28.4 \pm 20.3$ & 0.000 \\
\hline
\end{tabular}

Values are given as means \pm SD or $\mathrm{n}(\%) .{ }^{1}$ Defined by SCUAD.

Table 5. Predictive factors for SCUAD

\begin{tabular}{lll}
\hline Covariates & OR & $95 \%$ CI \\
\hline Fever & 3.966 & $1.636-9.614$ \\
Respiratory disease & 1.507 & $1.004-2.260$ \\
Ocular antihistamine & 1.841 & $1.153-2.938$ \\
Ocular symptoms & 2.370 & $1.469-3.825$ \\
\hline Mild intermittent AR ${ }^{1}$ & 0.285 & $0.150-0.544$ \\
Nasal obstruction & 0.454 & $0.283-0.726$ \\
Nasal pruritus & 0.574 & $0.377-0.874$ \\
\hline
\end{tabular}

${ }^{1}$ Mild intermittent vs. severe/moderate persistent.

tative of the patient population seen by GPs in France. Therefore, some patients may have presented with nonAR, but clusters were similar in patients with or without a diagnosis of allergy. Allergy was defined using broad criteria including skin tests and serum allergen-specific IgE. However, since the difference between in vivo and in vitro tests is not clear [33], it appears that the diagnosis of allergy is acceptable and representative of daily practice in primary care. However, the characterization of patients is not as precise as in specialist clinics. Previ- ous studies carried out by our group found that the ARIA classification performed in primary care or specialist practices in France was comparable [34, 35]. There were no major differences in phenotypes between patients with an allergy diagnosis and those without [34]. Moreover, the clinical results of the study previously published strongly suggest that the classification of patients was appropriate $[26,36]$.

Clusters may differ from the general population since patients usually consult for moderate-to-severe AR [34]. Moreover, it is possible that clusters carried out in specialist practices may differ, but this can only be studied in a specifically designed experiment.

This study was carried out in France but results may have been different if carried out in other countries.

\section{Strengths}

The present study is a real-life study without any exclusion criteria and is representative of the French patients seen by GPs. It has strengths including: (1) a sufficient power to detect changes, (2) a clinical validation of the cluster analysis during a follow-up of 14 days, this duration of treatment being sufficient to detect changes in AR [37-39], and (3) a comparison of clinical outcomes during treatment with the most widely used classification 
of patients (ARIA) [40]. Another strength of the study was that complexity in phenotype analysis may not be needed to understand AR in clinical practice. However, this finding should be replicated. To the best of our knowledge, this is the first study to assess cluster analysis in rhinitis.

\section{Generalizability}

In the present study, it was found that GPs treat all patients similarly whatever the cluster or ARIA class, confirming other European cohorts [41, 42]. Most patients received a combination of oral $\mathrm{H}_{1}$-antihistamines and intranasal corticosteroids. Physicians in real-life practice prescribe medication regardless of the phenotype and severity $[41,43]$. This is possibly because most patients present severe symptoms despite a treatment. The treatment prescribed is, however, at variance from guidelines.

Integrated care pathways (ICPs) [44] promote the translation of guidelines into local protocols and their subsequent application to clinical practice. ICPs differ from practice guidelines and need to record variations from planned care [45]. Any alteration to the practice identified within this ICP must be noted as a variance [46]. In the present study, variance was found for the treatment of patients by primary care physicians whereas the ARIA classification was reinforced. This variance analysis should be used to propose novel ICPs since, for the majority of patients, the practice differs from the guideline.
Surprisingly, the hypothesis-driven ARIA classification was more predictive of SCUAD than the data-driven cluster analysis. Patients with mild intermittent rhinitis were unlikely to be SCUAD patients. The data-driven phenotyping of the present study is unlikely to improve the management of patients with AR.

The present study questions whether data-driven clusters are needed in the management of AR in clinical practice. A recent study in chronic obstructive pulmonary disease did not detect any further interest, showing that the two clusters predicting mortality fit quite well with those that have been known for decades [47]. It is therefore possible that in relatively well-defined diseases, cluster analysis is not necessary to stratify patients. Moreover, the phenotypes resulting from cluster analysis should be validated prospectively with regards to the patient's outcomes. However, in the present study, clusters depended mainly on the symptoms and scores. This is probably due to the limited variables collected at baseline and to the study design. More studies are needed, but, to date, the clinical evaluation of patients appears to be sufficient for predicting uncontrolled patients during treatment and managing AR.

In conclusion, although an insufficient number of primary care physicians stratify patients in terms of severity, clinical parameters readily available in practitioners' offices are helpful in assessing the response to treatment in AR: cluster analysis in AR does not appear to have a relevant impact on disease stratification and clinical outcomes after treatment.

\section{References}

$>1$ Bousquet J, Anto JM, Sterk PJ, Adcock IM, Chung KF, Roca J, et al: Systems medicine and integrated care to combat chronic noncommunicable diseases. Genome Med 2011;3:43.

$>2$ Anderson GP: Endotyping asthma: new insights into key pathogenic mechanisms in a complex, heterogeneous disease. Lancet 2008; 372:1107-1119.

$>3$ Bousquet J, Anto JM, Demoly P, Schunemann HJ, Togias A, Akdis M, et al: Severe chronic allergic (and related) diseases: a uniform approach - a MeDALL-GA2LEN-ARIA position paper. Int Arch Allergy Immunol 2012; 158:216-231.

$>4$ Bousquet J, Khaltaev N, Cruz AA, Denburg J, Fokkens WJ, Togias A, et al: Allergic Rhinitis and Its Impact on Asthma (ARIA) 2008 update (in collaboration with the World Health Organization, GA(2)LEN and AllerGen). Allergy 2008;63(suppl 86):8-160.

$>5$ Bousquet PJ, Combescure C, Neukirch F, Klossek JM, Mechin H, Daures JP, et al: Visual analog scales can assess the severity of rhinitis graded according to ARIA guidelines. Allergy 2007;62:367-372.

-6 Simola M, Malmberg H: Sensation of nasal airflow compared with nasal airway resistance in patients with rhinitis. Clin Otolaryngol 1997;22:260-262.

7 Bousquet J, Lund VJ, Van Cauwenberge P, Bremard-Oury C, Mounedji N, Stevens MT, et al: Implementation of guidelines for seasonal allergic rhinitis: a randomized controlled trial. Allergy 2003;58:733-741.

$>8$ Numminen J, Ahtinen M, Huhtala H, Rautiainen M: Comparison of rhinometric measurements methods in intranasal pathology. Rhinology 2003;41:65-68.

$>9$ Senti G, Vavricka BM, Graf N, Johansen P, Wuthrich B, Kundig TM: Evaluation of visual analog scales for the assessment of symptom severity in allergic rhinoconjunctivitis. Ann Allergy Asthma Immunol 2007;98:134138.

10 Ciprandi G, Contini P, Pistorio A, Murdaca G, Puppo F: Sublingual immunotherapy re- duces soluble HLA-G and HLA-A,-B,-C serum levels in patients with allergic rhinitis. Int Immunopharmacol 2009;9:253-257.

11 Marseglia GL, Cirillo I, Klersy C, Caimmi D, Caimmi S, Castellazzi AM, et al: Clinical assessment of nasal decongestion test by VAS in adolescents. Pediatr Allergy Immunol 2009; 20:187-191.

12 Bousquet J, Bachert C, Canonica GW, Casale TB, Cruz AA, Lockey RJ, et al: Unmet needs in severe chronic upper airway disease (SCUAD). J Allergy Clin Immunol 2009;124: 428-433.

13 Bousquet PJ, Bachert C, Canonica GW, Casale TB, Mullol J, Klossek JM, et al: Uncontrolled allergic rhinitis during treatment and its impact on quality of life: a cluster randomized trial. J Allergy Clin Immunol 2010;126: 666-668.e1-e5.

14 Milligan G: Cluster analysis; in Kotz S, Campbell B, Balakrishnan N, Vidovic B (eds): Encyclopedia of Statistical Sciences. New York, Wiley, 1998. 
15 Barabasi AL, Gulbahce N, Loscalzo J: Network medicine: a network-based approach to human disease. Nat Rev Genet 2011;12:5668.

$\checkmark 16$ Anto JM, Pinart M, Akdis M, Auffray C, Bachert C, Basagana X, et al: Understanding the complexity of IgE-related phenotypes from childhood to young adulthood: a Mechanisms of the Development of Allergy (MeDALL) seminar. J Allergy Clin Immunol 2012;129:943-954.e4.

$\checkmark 17$ Henderson J, Granell R, Heron J, Sherriff A, Simpson A, Woodcock A, et al: Associations of wheezing phenotypes in the first 6 years of life with atopy, lung function and airway responsiveness in mid-childhood. Thorax 2008; 63:974-980.

18 Haldar P, Pavord ID, Shaw DE, Berry MA, Thomas M, Brightling CE, et al: Cluster analysis and clinical asthma phenotypes. Am J Respir Crit Care Med 2008;178:218-224.

-19 Simpson A, Tan VY, Winn J, Svensen M, Bishop CM, Heckerman DE, et al: Beyond atopy: multiple patterns of sensitization in relation to asthma in a birth cohort study. Am J Respir Crit Care Med 2010;181:12001206.

20 Clarisse B, Demattei C, Nikasinovic L, Just J, Daures JP, Momas I: Bronchial obstructive phenotypes in the first year of life among Paris birth cohort infants. Pediatr Allergy Immunol 2009;20:126-133.

-21 Just J, Gouvis-Echraghi R, Rouve S, Wanin S, Moreau D, Annesi-Maesano I: Two novel, severe asthma phenotypes identified during childhood using a clustering approach. Eur Respir J 2012;40:55-60.

22 Just J, Gouvis-Echraghi R, Couderc R, Guillemot-Lambert N, Saint-Pierre P: Novel severe wheezy young children phenotypes: boys atopic multiple-trigger and girls nonatopic uncontrolled wheeze. J Allergy Clin Immunol 2012;130:103-110.e8.

23 Newcomer SR, Steiner JF, Bayliss EA: Identifying subgroups of complex patients with cluster analysis. Am J Manag Care 2011; 17:e324-e332.

-24 Overall JE, Gibson JM, Novy DM: Population recovery capabilities of 35 cluster analysis methods. J Clin Psychol 1993;49:459-470.

25 Demoly P, Bousquet P, Mesbah K, Bousquet J, Devillier P: Visual analogue scale in patients treated for allergic rhinitis: an observational prospective study in primary care. Clin Exp Allergy 2013;43:881-888.
26 Bousquet PJ, Demoly P, Devillier P, Mesbah $\mathrm{K}$, Bousquet J: Impact of allergic rhinitis symptoms on quality of life in primary care. Int Arch Allergy Immunol 2013;160:393-400.

27 Suzuki Y, Kitazawa Y, Araie M, Yamagami J, Yamamoto T, Ishida K, et al: Mathematical and optimal clustering of test points of the central 30-degree visual field of glaucoma. J Glaucoma 2001;10:121-128.

28 Woolston A, Tu YK, Baxter PD, Gilthorpe MS: A comparison of different approaches to unravel the latent structure within metabolic syndrome. PloS One 2012; 7:e34410.

29 Moore WC, Bleecker ER, Curran-Everett D, Erzurum SC, Ameredes BT, Bacharier L, et al: Characterization of the severe asthma phenotype by the National Heart, Lung, and Blood Institute's Severe Asthma Research Program. TJ Allergy Clin Immunol 2007; 119:405-413.

-30 Kellberger J, Dressel H, Vogelberg C, Leupold W, Windstetter D, Weinmayr G, et al: Prediction of the incidence and persistence of allergic rhinitis in adolescence: a prospective cohort study. J Allergy Clin Immunol 2012;129: 397-402.e1-e3.

-31 Ballardini N, Kull I, Lind T, Hallner E, Almqvist C, Ostblom E, et al: Development and comorbidity of eczema, asthma and rhinitis to age 12: data from the BAMSE birth cohort. Allergy 2012;67:537-544.

-32 Shaaban R, Zureik M, Soussan D, Neukirch C, Heinrich J, Sunyer J, et al: Rhinitis and onset of asthma: a longitudinal population-based study. Lancet 2008;372:1049-1057.

33 Demoly P, Romano A, Bousquet J: In vivo methods for the study of allergy: skin tests; in Adkinson NF Jr, Bochner BS, Busse WW, Holgate ST, Lemanske RF Jr, Simons FER (eds): Middleton's Allergy, Principles and Practice, ed 7. Philadelphia, Elsevier, 2008, pp 1267-1280.

- 34 Bousquet J, Neukirch F, Bousquet PJ, Gehano P, Klossek JM, Le Gal M, et al: Severity and impairment of allergic rhinitis in patients consulting in primary care. J Allergy Clin Immunol 2006;117:158-162.

35 Bousquet J, Annesi-Maesano I, Carat F, Leger D, Rugina M, Pribil C, et al: Characteristics of intermittent and persistent allergic rhinitis: DREAMS study group. Clin Exp Allergy 2005;35:728-732.

36 Demoly P, Bousquet PJ, Mesbah K, Bousquet J, Devillier P: Visual analogue scale in patients treated for allergic rhinitis: an observational prospective study in primary care: asthma and rhinitis. Clin Exp Allergy 2013;43:881888 .

37 Fokkens WJ, Jogi R, Reinartz S, Sidorenko I, Sitkauskiene B, van Oene C, et al: Once daily fluticasone furoate nasal spray is effective in seasonal allergic rhinitis caused by grass pollen. Allergy 2007;62:1078-1084.

38 Bousquet J, Bachert C, Canonica GW, Mullol $\mathrm{J}$, Van Cauwenberge P, Bindslev Jensen C, et al: Efficacy of desloratadine in intermittent allergic rhinitis: a GA(2)LEN study. Allergy 2009;64:1516-1523.

39 Bousquet J, Bachert C, Canonica GW, Mullol J, Van Cauwenberge P, Jensen CB, et al: Efficacy of desloratadine in persistent allergic rhinitis - a GA(2)LEN study. Int Arch Allergy Immunol 2010;153:395-402.

-40 Bousquet J, Schunemann HJ, Samolinski B Demoly P, Baena-Cagnani CE, Bachert C, et al: Allergic Rhinitis and its Impact on Asthma (ARIA): achievements in 10 years and future needs. J Allergy Clin Immunol 2012;130: 1049-1062.

-41 Ramirez LF, Urbinelli R, Allaert FA, Demoly $P$ : Combining $\mathrm{H}_{1}$-antihistamines and nasal corticosteroids to treat allergic rhinitis in general practice. Allergy 2011;66:1501-1502.

42 Valero A, Ferrer M, Baro E, Sastre J, Navarro AM, Marti-Guadano E, et al: Discrimination between moderate and severe disease may be used in patients with either treated or untreated allergic rhinitis. Allergy 2010;65:16091613.

43 del Cuvillo A, Montoro J, Bartra J, Valero A, Ferrer M, Jauregui I, et al: Validation of ARIA duration and severity classifications in Spanish allergic rhinitis patients - the ADRIAL cohort study. Rhinol 2010;48:201-205.

44 Campbell H, Hotchkiss R, Bradshaw N, Porteous M: Integrated care pathways. BMJ 1998; 316:133-137.

45 How to produce and evaluate an integrated care pathway (ICP): information for staff. Great Ormond Street Hospital for Children. 2010. www.gosh.nhs.uk.

46 Integrated Care Pathways Users in Scotland (ICPUS). A workbook for people starting to develop integrated care pathways. http:// www.icpus.org.uk2007.

47 Burgel PR, Paillasseur JL, Peene B, Dusser D, Roche N, Coolen J, et al: Two distinct chronic obstructive pulmonary disease (COPD) phenotypes are associated with high risk of mortality. PloS One 2012;7:e51048. 